“(C) 2018 IEEE. Personal use of this material is permitted. Permission from IEEE must be obtained for all other uses, in any current or future media, including reprinting/republishing this material for advertising or promotional purposes, creating new collective works, for resale or redistribution to servers or lists, or reuse of any copyrighted component of this work in other works." 


\title{
Anticipating industry convergence in the context of Industry 4.0
}

\author{
Svetlana Okara ${ }^{1}$, Stefanie Broering ${ }^{2}$, Nathalie Sick ${ }^{3}$ \\ ${ }^{1}$ Institute of Business Administration at the Department of Chemistry and Pharmacy, University of Muenster, Germany \\ ${ }^{2}$ Institute for Food and Resource Economics, University of Bonn, Germany \\ ${ }^{3}$ School of Systems, Management and Leadership, Faculty of Engineering and IT, UTS, Sydney, Australia
}

\begin{abstract}
The merger of the digital and physical world in the context of Industry 4.0 is about to disrupt value chains and markets in almost every industry sector. In this context, the Internet of Things (IoT), enabling linkages and communication between physical and virtual objects, is the technological foundation of implementing Industry 4.0. In such a fast-paced environment, it is vital for companies to react quickly and exploit new business opportunities. One critical example is the interplay between logistics and information and communications technology (ICT) industries, where IoT has the potential to align goods and information flows in an unprecedented manner. The arising new functionalities, services and products show potential to blur the industries' boundaries and give birth to a whole new industry segment. Therefore, the present study strives to anticipate industry convergence between logistics and ICT industries in the realm of IoT. The empirical patent analysis is based on IPC coclassification and assignee structure. The analyses are refined along the different levels of IoT to provide detailed insights for companies where new technological and market competences need to be acquired.
\end{abstract}

\section{INTRODUCTION}

Nowadays, about two billion people communicate via the internet. While this number grows steadily, the next big leap in development will contain the implementation of the internet in machines and intelligent objects to simplify their communication and coordination [[1]]. The merging of the digital and the physical world within the framework of the fourth industrial revolution, called Industry 4.0, will formatively change whole industries, markets and value chains. Companies will have to face and adapt to these challenges regardless of their current success and have to reorient themselves in order to ensure their competitive advantage and succeed within their future market positioning. An early and rapid response towards these upcoming changes is crucial to remain competitive and generate new business opportunities [[2]].

At the center of technological developments within Industry 4.0 is the Internet of Things (IoT), which describes the networking and communication between physical and virtual objects [[3]]. In such a fast-paced environment, it is vital for companies to react quickly and exploit new business opportunities. One critical example is the interplay between logistics and information and communications technology (ICT) industries, where IoT has the potential to align goods and information flows in an unprecedented manner [[4]]. Logistics have already been using ICT for many years to optimize the flow of information. However, IoT could bring the interplay of logistics and ICT technologies to a whole new level by combining physical assets and virtual flows of information. The arising new functionalities, services and products show potential to blur the industries' boundaries and give birth to a completely new industry segment. In such a setting of industry convergence, it is vital for companies to anticipate a convergence process at a very early stage to focus their innovation activities and generate a competitive edge [[5]]. With changing technological and market environments in the context of convergence, there is a great deal of uncertainty for affected companies as they can no longer rely on their core competences. With the emergence of a new inter-industry segment, companies often lack the necessary experience and knowledge on new technologies and markets. Hence, companies need to define effective innovation strategies and find collaboration partners to minimize the emerging skill gaps [[6]]. To provide the basis for these strategic alignments, the target of this work is to anticipate and assess convergence developments at the interface of logistics and ICT industry by answering the following research question: Are there signs of convergence between the logistics and ICT industry in the area of IoT?

Patent analyses, more specifically IPC co-classification and assignee analysis, are used to anticipate convergence movements. The analyses are refined along the different levels of IoT to provide detailed insights for companies where new technological and market competences need to be acquired. The findings can be used as a basis to craft innovation strategies and identify suitable collaboration partners to close technology and market competence gaps in the context of Industry 4.0.

\section{THEORETICAL BACKGROUND}

Convergence is a young field of research that has grown significantly in the last twenty years. The concept of convergence in the economic context dates back to Nathan Rosenberg (1963). He coined the term in the context of technological developments in the machine tool industry in the second half of the 19th century. More specifically, he delineated the term "technological convergence" of "sequences of parallel and unrelated activities" and described the approach of unrelated industries on a technological level [[7]]. In recent years, the phenomenon has been discussed primarily in the context of the ICT and multimedia industries. Despite the widespread use in literature, there is to date no common definition of convergence. Many sources refer to the blurring of 
industrial boundaries and increasing overlaps in technologies and services. Curran and Leker (2011) define convergence as: "Blurring of boundaries between at least two hitherto disjoint areas of science, technology, markets, or industries. Through this convergence, a new (sub-) segment is being created in a new spot as a merger of (parts) of the old segments. "[[5]]

It is vital for companies to anticipate such convergence processes at a very early stage to be able to react accordingly to sometimes earth-shattering developments. Therefore, scholars have developed models of convergence to characterize and anticipate convergence processes. Hacklin (2010) describes convergence as an evolutionary process spanning four consecutive phases - knowledge, technology, application and industry convergence [[8]]. Curran and Leker (2011) suggest the convergence process depicted in Fig. 1. as an idealized time series of events, starting with convergence on the scientific level, moving to the technology and market level, and finally ending at the industry level [[9]].

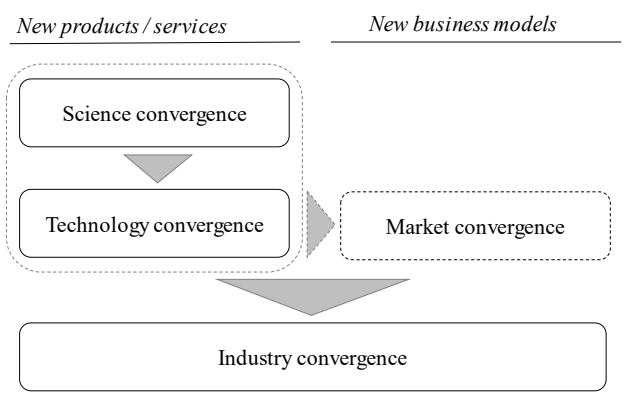

Figure 1: Sequential process of industry convergence [5].

Scientific convergence can be observed by an increase in interdisciplinary citations and research collaborations between hitherto different fields of science. As the distance between the science fields decreases, applied science and technology developments follow, resulting in technology convergence. In the next step, a market convergence can be triggered, which manifests itself in new product- or service-marketcombinations. The final step is industry convergence as a new inter-industry segments emerges. While convergence in science- and technology-intensive industries goes through all four steps, market convergence can be triggered by new business models without the prior steps, leading directly to industry convergence [[9]].

\section{INTERNET OF THINGS AS A MAIN DRIVER OF INDUSTRY 4.0}

Technological developments, and particularly digital technologies around Industry 4.0, are one of the main drivers of converging industries. The merger of the digital and physical world in the context of Industry 4.0 is about to disrupt value chains and markets in almost every industry sector. The term was coined in 2011 by the German government within the framework of a high-tech strategy for Germany [[9]]. The term Industry 4.0 refers to the previous three stages of industrial revolution. The first industrial revolution began with the invention of the steam engine and the introduction of mechanical production at the end of the 18th century. This was followed by the second industrial revolution with electricdriven mass production and division of labor from the end of the 19th century. It was replaced by the use of electronics and IT to further automate production as part of the third industrial revolution from about 1970 onwards [[3]]. While the third industrial revolution focused on the automation of individual machines and processes, Industry 4.0 connects the entire value chain through an end-to-end digitization to network all physical assets [[11]]. With the help of autonomous communication of technologies and devices, a new level of organization and control of value chains is to be achieved. This offers companies considerable potential for process optimization and new business opportunities [[9]].

The technological possibilities to link resources, information, objects and people is the basis of these developments. While the first three industrial revolutions were triggered by mechanization, electricity and IT, the Internet of Things (IoT), enabling linkages and communication between physical and virtual objects, is the technological foundation of Industry 4.0 [[3]]. A clear and generally accepted definition of IoT is not yet available. The term was already used in the early 2000 s as part of MIT AutoID Labs, a network of academic researchers concerned with the field of Radio Frequency Identification Technology (RFID) [[12]]. While the focus was then on the identification and tracking of objects, the term today has a much wider range. Sundmaeker et al. (2010) talk about IoT as:

„Dynamic global network infrastructure [...] where
physical and virtual 'things' [...] are seamlessly
integrated into the information network." [[13]]

It is clear that not only virtual "things" are connected through the IoT, but also physical objects are networked and included in the global information network. Atzori, Iero and Morabito (2010) take a closer look at the "things" or objects and describe the idea behind IoT with:

\section{„Pervasive presence around us of a variety of things or objects [...] which, through unique addressing schemes, are able to interact with each other and cooperate with their neighbors to reach common goals". [[12]]}

Trappey et al. (2017) proposed a systematization of IoT containing four levels (Fig. 2). The IoT is based on a combination of technologies that can be allocated to each level [[14]].

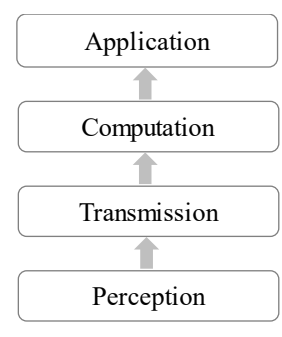

Figure 2: IoT-layers [15]. 
The first level perception deals with sensors and actuators for perception and data acquisition. The purpose of this level is to provide physical objects with sensory benefits such as seeing, hearing, smelling, and sensing. The next level transmission transfers the information gained on the perception level to higher levels. Data transmission is governed by protocols and depends on factors such as power, range and storage capacity. The third level computation is used for data processing and decision-making and contains components such as hardware, software and algorithms. The fourth and last level application uses the transmitted information from previous levels for tailored applications, for example in the production, health or logistics sector [[15]].

\section{DATA AND METHODS}

In the following we draw upon patent analysis as it is an established measure to assess processes of technological convergence and thus anticipate convergence. In a first step, patents in the area of IoT are identified. To cover just the area of IoT without any industry or application focus, the identification of suitable patents is based on the first three layers of IoT while leaving out the application level. For this purpose, a combination of patent classifications (IPC classes) and key words is used to extract a dataset which allows for the highest possible quality of results (Table I).

Table I: Search term to identify patents in the area of IoT.

\begin{tabular}{|c|c|c|c|}
\hline IoT-layer & IPC-Classes & \multicolumn{2}{|c|}{ Keywords } \\
\hline Perception & H04L12/24 & $\begin{array}{l}\text { Sensor, } \\
\text { actuator, RFID, }\end{array}$ & \multirow{10}{*}{$\begin{array}{l}\text { Internet of } \\
\text { Things (IoT) } \\
\text { Cyber-physical } \\
\text { system (CPS) } \\
\text { Machine to } \\
\text { machine } \\
\text { (M2M) }\end{array}$} \\
\hline \multirow{6}{*}{ Transmission } & H04L29/08 & \multirow{6}{*}{ Protocol } & \\
\hline & H04L29/06 & & \\
\hline & H04L12/28 & & \\
\hline & H04L29/12 & & \\
\hline & H04L12/56 & & \\
\hline & H04L29/02 & & \\
\hline \multirow{3}{*}{ Computation } & H04L12/26 & \multirow{3}{*}{ Compute } & \\
\hline & G06F15/16 & & \\
\hline & G06F13 & & \\
\hline
\end{tabular}

The patent data were collected using PatBase ${ }^{\circledR}$, a professional patent database that provides access to more than 100 million documents from over 100 countries worldwide [[16]]. It covers more than 56 million patent families and offers all search and analysis functions. The patents are grouped into extended patent families, avoiding duplication. Since IoT is a very recent field of research, the investigation period covers ten years from 2005 to 2014 [[17]]. The investigation period ends with the year 2014 due to the delay of 18 months between application and publication. Thereby, we use the patent application date as the earliest possible indicator for the appearance of a new technology [[18]].

To allow for comparisons of past and recent developments, the sample is split up into two sets of data, covering the period 2005-2009 and 2010-2014.

Although the IoT term was coined in the early 2000s, its evolution into a comprehensive research object happened at a lower pace. The same holds true for its dissemination into the public. Only in 2005, IoT occasionally appeared in the mass media and was featured in a report by the UN International Telecommunications Union. IoT made its first breakthrough in 2008 when it appeared in the list of six "Disruptive Civil Technologies" of the US National Intelligence Council. The launch of the iPhone in 2007 and the consequent growth of internet-connected devices such as iPhones, tablets and iPads also contributed to the development of IoT in the coming years. In 2011, companies such as Cisco and IBM initiated major marketing and education initiatives in the new technology area [[17]]. It thus becomes clear that IoT technology started to develop and grow in the period 2005-2009 and gained much more prominence between 2010 and 2014.

In a second step, an analysis of the most active patent assignees from both industries enriches the findings of the IPC coclassification. To this end, the patent portfolio of the top ten logistics companies with the highest turnover in the periods 2005-2009 and 2010-2014 are examined [[22]]. Firstly, the shares of patents in leading ICT classes are calculated and compared between the two data sets. Secondly, the shares of patents are calculated at the respective IoT levels and also compared in both periods.

In a third step, an IPC co-classification analysis is applied to determine technological convergence and thus anticipate possible industry convergence in the field of IoT between logistics and ICT. The IPC classes representing the logistics and ICT industry are based on the catchword index in WIPO and Espacenet as well as the "Technology Classification" proposed by WIPO (Table II) [[19], [20], [21]].

Table II: Assignment of logistics and ICT technologies to IPC classes.

\begin{tabular}{|c|c|c|}
\hline Area & Technologies & $\begin{array}{c}\text { IPC- } \\
\text { Classes }\end{array}$ \\
\hline \multicolumn{3}{|c|}{ Logistics } \\
\hline \multirow{2}{*}{$\begin{array}{l}\text { Data processing in } \\
\text { logistics }\end{array}$} & $\begin{array}{l}\text { Data processing systems and } \\
\text { procedures for logistics }\end{array}$ & G06Q50/28 \\
\hline & $\begin{array}{l}\text { Administration / management of } \\
\text { logistics }\end{array}$ & G06Q10/08 \\
\hline \multirow{3}{*}{$\begin{array}{l}\text { Promotion and } \\
\text { storage }\end{array}$} & Packing machines and processes & B65B \\
\hline & Transport and storage devices & B65G \\
\hline & $\begin{array}{l}\text { Containers for storage or } \\
\text { transportation }\end{array}$ & B65D \\
\hline \multirow{2}{*}{ Post } & Franking machines & G07B17 \\
\hline & Sorting of mail & B07C3 \\
\hline Transport logistics & Traffic control & G08G \\
\hline
\end{tabular}




\begin{tabular}{|c|c|c|}
\hline & Navigation in the road network & G01C21/26 \\
\hline & Route planning & $\mathrm{G} 01 \mathrm{C} 21 / 34$ \\
\hline \multicolumn{3}{|c|}{ ICT } \\
\hline \multirow{2}{*}{$\begin{array}{l}\text { Computer } \\
\text { technology }\end{array}$} & Electrical digital data processing & G06F \\
\hline & Speech recognition and analysis & G10L \\
\hline Wireless & Wireless communication & $\mathrm{H} 04 \mathrm{~W}$ \\
\hline Digital & Transmission of digital & H04L \\
\hline \multirow{5}{*}{ Telecommunication } & Transmission systems for signals & G08C \\
\hline & Telephone traffic & $\mathrm{H} 04 \mathrm{M}$ \\
\hline & Dial technologies & H04Q \\
\hline & $\begin{array}{l}\text { Transmission of information } \\
\text { signals }\end{array}$ & H04B \\
\hline & Broadcasting & $\mathrm{H} 04 \mathrm{H}$ \\
\hline
\end{tabular}

\section{FINDINGS AND DISCUSSION}

\section{A. Developments of patenting activties in IoT}

In total, 235,176 patent families representing IoT were identified. Data set I with IoT patents for the period 2005-2009 comprises 110,263 patent families, while data set II contains 124,913 patent families for the period 2010-2014. This represents a growth of $13 \%$, which reflects the progressive development of the technology. Nevertheless, it can be expected that the positive trend in patents between the two periods would have increased by more than $13 \%$, as IoT experienced a significant growth spurt between 2010 and 2014 and was included in many companies' innovation programs [[17]]. An explanation for this can be the deeper consideration of the individual IoT levels. Fig. 3 shows that patents at the perception and transmission levels have increased significantly by $64 \%$ and $34 \%$, while patents at the computation level have decreased by $13 \%$. It suggests that innovation activities in the IoT area focus primarily on the perception and transmission levels. These findings are confirmed in several studies, which show that sensors and actuators as perception technologies as well as RFID and digital data transmission represent the central drivers and components of IoT on behalf of the transmission level [[12]].

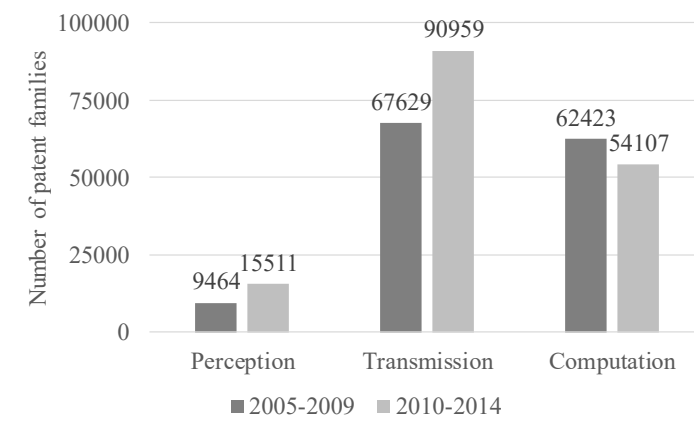

Figure 3: Number of patents according to IoT level.
Although patents concerning computation make up a large part of the dataset, their evolution is declining. This may suggest that, while the layer has a high weight in the IoT area, it offers less room for further innovation in the IoT context. The interplay of hardware and software as part of the computation level is the traditional core of the computer industry and could explain its importance and its decline compared to emerging technologies [[24]]. Perception patents have the lowest number and largest growth compared to the other two levels, suggesting the emergence of new technologies in this area.

\section{B. Assignee analysis}

For further analysis of the IoT area, the assignees with the most patent applications are examined in more detail. The identification of the Top 10 patent applicants in Fig. 4 over the period 2010-2014 shows that they all belong to the ICT industry. In addition, they account for almost $25 \%$ of the patents in the dataset. This indicates, on the one hand, that IoT is primarily located in the area of expertise of ICT companies. On the other hand, the distribution shows that there are several large ICT players engaged in R\&D in the IoT area.

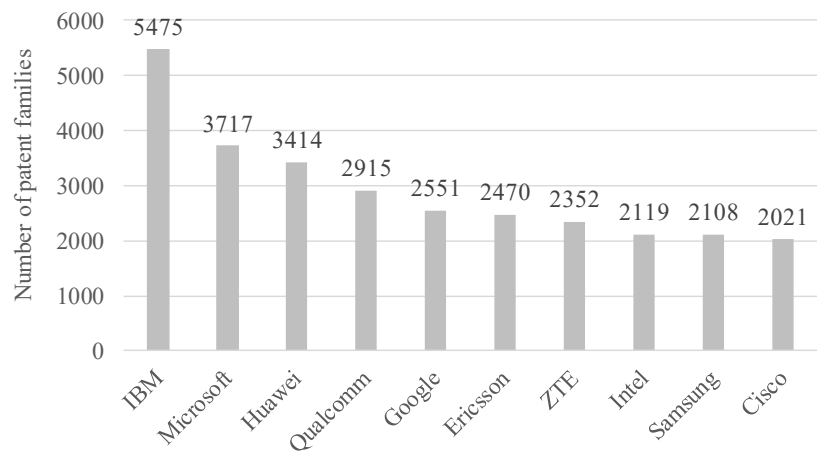

Figure 4: Top 10 patent assignees in IoT 2009-2014.

While ICT companies are dominating IoT R\&D, logistics companies are weakly represented. The top ten largest logistics companies account for only 30 patents between 2010 and 2014 in the IoT area, most of which are attributable to UPS, Deutsche Post DHL Group and FedEx [[22]]. One reason for this discrepancy is the fact that the ICT industry is a traditionally very patent-rich industry, due to its high technological focus [[25]]. However, in the field of logistics, process innovation plays an important role, whereby process innovations are less likely to be patented than product innovations [[26]]. As a result, the logistics industry in general can be expected to have a lower propensity to patent than the ICT industry [[25]]. Nevertheless, the considerable difference in the number of patents shows that logistics companies in the IoT field are significantly underrepresented compared to ICT companies. Comparing the assignees from data sets I and II draws a similar picture with regard to the composition of the ten leading patent assignees. Nokia, Sony and Fujitsu are among the top 10 in 2005-2009, while replaced by Google, Intel and ZTE in 20102014. Google has the largest patent growth, registering twice as many patents in 2010-2014 as in the previous five years. A look at other technology companies such as Amazon and Facebook 
shows a similar development. In the period 2010-2014 Amazon quintuples its applications and ends up with 1,240 patents among the top 20 Assignees. With 741 applications, Facebook generates three times as many patents as $2005-2009$, bringing the company closer to the top 20 . None of the ICT companies has a comparable increase in patents, while in some cases the patent volume is even declining. These developments coincide with the rapid growth of technology companies such as Google, Amazon and Facebook [[27]] and show that these companies are establishing themselves as strong competitors to traditional ICT and logistics companies in the IoT area.

\section{IPC co-classification}

IPC co-classification is used to assess the interconnectedness of the logistics and ICT industries in IoT at a technological level, i.e. technology convergence. Dataset I includes 902 patents that are classified as logistics patents, while in dataset II the number increases to 1,299 logistics patents. When identifying ICT patents, it is natural that all IoT patents contain IPC classes in the ICT industry, as IoT patent classes make up a subset of ICT patent classes. Table III shows that the proportion of logistics patents, which are also classified as ICT patents, amounts to $100 \%$ in both data sets. ICT patents, which are simultaneously classified as logistics patents, amount to $0.82 \%$ in data set I and $1.04 \%$ in data set II. With a share of only $0.82 \%$, the proportion of ICT patents co-classified as logistics is very low, suggesting that only a small proportion of ICT patents are related to logistics. Nevertheless, the increase to $1.04 \%$ shows that the number of co-classified patents is increasing, leading to the intertwining of technologies, albeit still at a very low level.

Table III: Co-Classification matrices for ICT and logistics in IoT.

\begin{tabular}{|c|c|c|}
\hline \multicolumn{3}{|c|}{ Co-Classification 2005-2009 } \\
\hline & ICT (N=110.263) & Logistics (N=902) \\
\hline ICT & - & $100 \%$ \\
\hline Logistics & $0,82 \%$ & - \\
\hline \multicolumn{3}{|c|}{ Co-Classification 2010-2014 } \\
\hline & ICT (N=124.913) & Logistics (N=1299) \\
\hline ICT & - & $100 \%$ \\
\hline Logistics & $1,04 \%$ & - \\
\hline
\end{tabular}

The claim that the integration of logistics and ICT technologies is progressing slowly is supported by an increasing trend of coclassifications of logistics patents in ICT (Fig. 5).

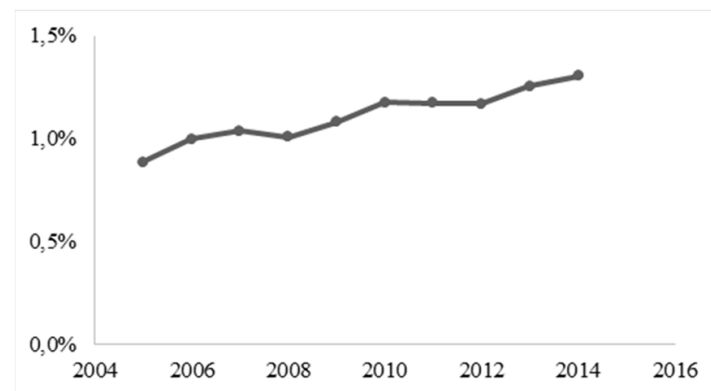

Figure 5: Development of ICT patents in IoT co-classified as logistics.

The development of logistics and ICT patent applications in the IoT area shown in Fig. 6 within a ten-year period shows a similar trend. In 2007, the first peak is reached in both industries, which can be linked to the breakthrough in IoT through the introduction of the iPhone in 2007. Despite the progressive development of IoT, patent applications in 2008 and 2009 decreases due to the global economic crisis. From 2010, an upwards trend is again to be seen, which is supported by the introduction of the new Internet Protocol IPV6, which substantially increases the IP address space and ensures a nationwide connection of devices to the Internet for the future [[28]]. In 2013 and 2014, the growth of logistics patents is progressing more rapidly than ICT patents.

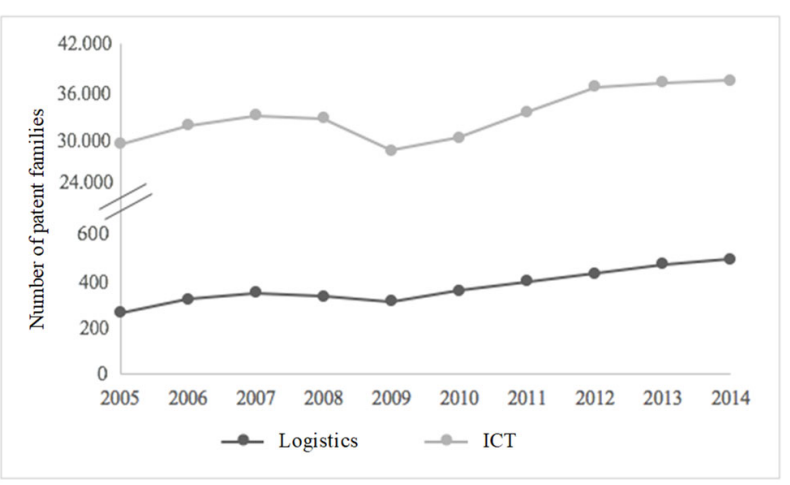

Figure 6: Development of logistics and ICT patenting avtivities in IoT.

In summary, IPC co-classifications do not reveal clear signs of convergence at this stage. The level of co-classifications is still low, whereas the rising trend may point to upcoming convergence tendencies. In addition, the relationship between logistics and ICT patents in IoT datasets is unbalanced. Conversely, while ICT seems to play an essential role in the IoT context for logistics, it is not the case vice versa. At this point it can be assumed that we rather observe a shift in value chains between both industries than a convergence process. On the other hand, new players such as Amazon are entering the field showing that IoT is likely to bring up new and complementary technologies, products, and services changing the logistics industry landscape [[29]]. The competition shifts from individual services to the establishment of complete productservice-systems and an optimized overall performance. It not only replaces established services and processes, but also adds new complementary functionalities that open markets for new 
services. At the interface between the ICT and logistics industry, new complementary functions and services are being created, for example in the form of smart inventory systems or digital logistics platforms. For example, Agheera, a provider of tracking solutions, has developed a platform that consolidates real-time data streams across multiple providers and telematics devices. In this way, customers can track their entire supply chain in real time [[30]]. Thus, new business models emerge that not only replace old logistics services with more innovative solutions, but also create new and complementary services at the interface between the ICT and logistics industries.

\section{IoT levels of analysis}

Having in mind these overall developments as well as the varying developments concerning the four levels of IoT (section 2), a more detailed look at the different levels of IoT at the interface between logistics and ICT is summarized in Table IV.

Table IV: Changes in co-classifications from 2005-2009 to 2010-2014 by IoT level.

\begin{tabular}{|c|c|c|c|c|}
\hline & IPC/level & Perception & Transmission & Computation \\
\hline $\begin{array}{c}\text { Data } \\
\text { processing I }\end{array}$ & G06Q50/28 & $-12 \%$ & $266 \%$ & $-31 \%$ \\
\hline $\begin{array}{c}\text { Data } \\
\text { processing II }\end{array}$ & G06Q10/08 & $22 \%$ & $98 \%$ & $13 \%$ \\
\hline $\begin{array}{l}\text { Transport } \\
\text { /Storage }\end{array}$ & B65B & $-12 \%$ & $24 \%$ & $6 \%$ \\
\hline Container & B65G & $121 \%$ & $5 \%$ & $-46 \%$ \\
\hline Packaging & B65D & $6 \%$ & $-14 \%$ & $-16 \%$ \\
\hline Franking & G08G & $13 \%$ & $46 \%$ & $-17 \%$ \\
\hline Post sorting & B07C3 & $-100 \%$ & $-12 \%$ & $-37 \%$ \\
\hline $\begin{array}{l}\text { Traffic } \\
\text { control }\end{array}$ & G07B17 & $-85 \%$ & $-37 \%$ & $-50 \%$ \\
\hline Navigation & G01C21/26 & $-56 \%$ & $8 \%$ & $13 \%$ \\
\hline Routing & G01C21/34 & $194 \%$ & $68 \%$ & $17 \%$ \\
\hline
\end{tabular}

\section{Perception}

Co-classifications in perception are lowest compared to other levels. The highest values are reached in connection with data processing in administration or management in logistics and traffic control. With regard to the change between the two periods, in particular increases of $121 \%$ in combination with transport and storage devices and of $194 \%$ with route planning are striking. Sensors that record data from the environment have long been used in traffic control, for example, to align the traffic light phases with the traffic volume. As part of IoT, sensor technologies are experiencing significant growth in transportation and storage devices, with sensors in warehouses being equipped with sensors to detect obstacles and move as autonomously as possible. These include, for example, intelligent forklifts from Swisslog and mobile warehouse robots from Amazon [[31]]. In the area of route planning, there are opportunities to equip vehicles with sensors so that algorithms can calculate optimized routes based on the collected information. In its On-Road Integrated Optimization and Navigation (ORION) project, for example, UPS collected all the data on routes, downtime, and safety with the help of GPS and sensors in vehicles, allowing ORION's advanced algorithm to significantly optimize trip planning [[32]].

\section{Transmission}

The transmission level contains the highest number of coclassifications, in particular in connection with G06Q50/28, G06Q10/08, G08G, G01C21/26 and G01C21/34. By far the highest increase is the overlap with G06Q50/28, followed by G06Q10/08 and G01C21/26. The results demonstrate the high importance of transmission technologies for IoT applications in logistics. The transfer of data is located between the upstream data acquisition and the downstream data processing and thus connects the perception and computation levels. The significantly increasing patenting activity in transmission technologies together with data processing in logistics and route planning is evidence of the increasing integration of logistics areas with IoT technologies. Data transmission is the essential function for data processing in logistics, which explains the high degree of interdependence of these areas. In addition, in transport logistics, the transmission of real-time information to track deliveries is of utmost importance [[33]].

The focus of the co-classifications clearly is on the patent classes H04L29/06 and H04L29/08. H04L29/06 is characterized by protocols that regulate data transmission at different levels using communication standards. H04L29/08 includes various methods for controlling data transmission. H04L29/06 shows the highest increases of co-classifications across all logistics areas except franking, while H04L29/08 only gains more interfaces in combination with data processing in logistics, traffic control and route planning. It can thus be concluded that data transmission protocols are the most important component of IoT for applications in logistics.

\section{Computation}

The number of co-classifications in computation is higher than at the perception level and lower than at the transmission level. The highest values are achieved at the interfaces to G06Q10/08, G08G, G01C21/26 and G01C21/34. With regard to relative changes, however, there are no overall upward trends observable as in the other levels. The number of coclassifications is decreasing and increases are low. On the one hand, the high level of co-classifications indicates a high relevance of computational-level technologies for logistics. On the other hand, the decreasing or nearly constant values imply that the focus in the context of IoT is not on the evolution of computation. This suggests that innovation in this area could be saturated, while the level remains essential for IoT applications. The progress in IoT related to logistics instead takes place in technologies related to perception and transmission. This in turn goes hand in hand with the findings and the studies of other authors [[12]]. 
Looking at the patent portfolio of the top ten largest logistics companies in Fig. 7 reveals that the patents in the IoT levels account for a small part of the total portfolio. The logistics companies do not undertake more R\&D at the perception level in 2010-2014 than in the previous five years. By contrast, there is a clear increase in transmission, while the number of patents decreases in computation. On the one hand, it can be seen that logistics companies are pursuing more R\&D in the field of data transmission. Analogous to previous findings, the computational level in the context of IoT seems to be losing importance for logistics. A discrepancy arises in relation to the perception level. According to the IPC co-classification analysis, it gains more and more relevance in IoT applications for logistics. Nevertheless, logistics companies do not seem to be strengthening R\&D in this area.

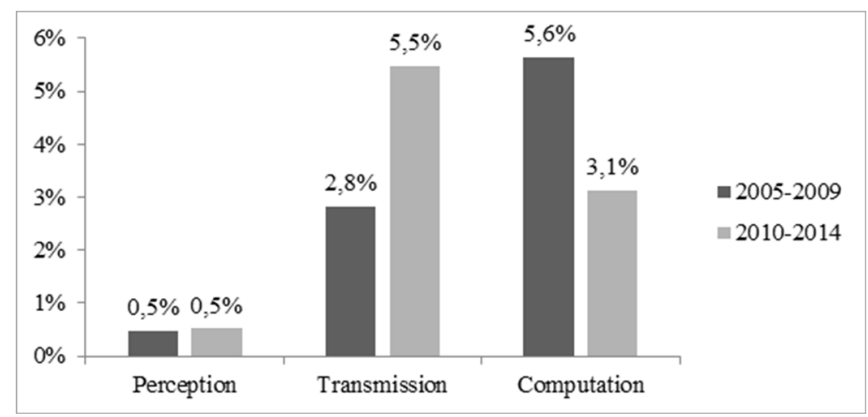

Figure 7: Shares of IoT levels in logistics companies.

\section{CONCLUSIONS}

The aggregated consideration of technological overlaps between the logistics and ICT industries in the area of IoT revealed no clear signs of convergence. While the low level of co-classifications provides no indication of potential convergence, it becomes clear that technological overlaps increase over time. The examination of individual coclassifications between logistics and ICT technologies also provides information on which logistics areas and ICT technologies are currently the most intertwined and which technologies are becoming increasingly important for logistics areas, namely electrical digital data processing, digital data transmission and wireless communication. A closer look at technological overlaps between the individual IoT levels and logistics implies that innovation activities take place mainly in data acquisition with the aid of sensors and actuators (perception level) and protocol-controlled data transmission (transmission level). Meanwhile, computing and decisionmaking technologies (computational level) offer less room for innovation.

Compared to ICT companies, established logistics companies in the IoT sector are clearly underrepresented. While the ICT industry will have to deviate less from the technological path it has taken so far, logistics companies can no longer rely on their core competencies and are confronted with technology-related skills gaps. ICT technologies of digital data transmission and wireless communication, which are becoming increasingly important in the context of IoT, occupy a small proportion of the established logistics companies' patent portfolios compared to traditional logistics areas such as storage or logistics processing. In addition, it becomes clear that logistics companies face competence gaps concerning perception-level technologies, even though these are crucial for IoT developments. Logistics already seems to be building competencies in the essential transmission level for IoT, but companies still need to close technology competence gaps in order to comprehensively integrate IoT in logistics processes. Notwithstanding these insights, the study faces several limitations. The use of patents and especially co-classifications to study technological convergence provides an adequate way to analyze technological interdependencies and their changes over time. Since IPC classes do not always delineate technology fields and, above all, newly emerging technologies, convergence developments can be additionally examined with the help of further methods [[34]]. A semantic analysis that measures similarities between patents from different technological fields would be conceivable. An increasing semantic similarity can be interpreted as an approximation of terminology and terminology and can be a sign of convergence. In addition, technological patterns can be visualized using patent maps by showing and comparing R\&D hotspots [[5]]. Furthermore, when interpreting the results, the discrepancy in the patenting behavior of the logistics and ICT industry must be taken into account, since research-intensive and technologyoriented industries (such as the ICT industry) traditionally have a considerably larger patent volume. In this respect, in order to anticipate industrial convergence, scientific convergence can be analyzed as well. By measuring the distance between scientific publications in the logistics and ICT industries, additional insights into a possible convergence of logistics and ICT industries can be gained.

\section{LITERATURE}

[1] D. Miorandi, S. Sicari, F. De Pellegrini, and I. Chlamtac, "Internet of things : Vision, applications and research challenges," Ad Hoc Networks, vol. 10, no. 7, pp. 1497-1516, 2012.

[2] A. Roth, Einführung und Umsetzung von Industrie 4.0, Berlin, Heidelberg: Springer, 2006.

[3] H. Kagermann, J. Helbig, A. Hellinger, W. Wahlster, Recommendations for implementing the strategic initiative INDUSTRIE 4.0: Securing the future of German manufacturing industry, Final report of the Industrie 4.0 Working Group, Forschungsunion, 2013.

[4] H. J. Bullinger, M. Hompel, "Internet der Dinge - www.internet-derdinge.de," Heidelberg: Springer, 2007.

[5] C. Curran and J. Leker, "Patent indicators for monitoring convergence examples from NFF and ICT," Technol. Forecast. Soc. Chang., vol. 78(2), pp. 256-273, 2011

[6] S. Bröring, "Developing innovation strategies for convergence - is ' open innovation' imperative?," International Journal of Technology Management, vol. 49 (1-3), pp. 272-294, 2010.

[7] N. Rosenberg, "Technological Change in the Machine Tool Industry, 1840-1910," The Journal of Economic History, vol. 23(4), pp. 414-443, December 1963.

[8] F. Hacklin, C. Marxt, F. Fahrni, "An evolutionary perspective on convergence: inducing a stage model of inter-industry innovation," International Journal of Technology Management, vol. 49(1-3), pp. 220249, 2010.

[9] N. Preschitschek, C. Curran, and J. Leker, "The Importance of Access to Resources in a Setting of Industry Convergence : The Case of Agriculture and Chemistry," Proceedings of PICMET'11 Technology Management in the Energy Smart World, Portland, 2011.

[10] J. Smit, S. Kreutzer, C. Moeller, and M. Carlberg, "Industry 4.0, Policy 
[11] PWC, "Industry 4.0: Building the digital enterprise," 2016, http://www.pwc.com/gx/en/industries/industry-4.0.html, retrieved $13 / 05 / 2017$.

[12] L. Atzori, A. Iera, and G. Morabito, "The Internet of Things : A survey," Comput. Networks, vol. 54(15), pp. 2787-2805, 2010.

[13] H. Sundmaeker, P. Guillemin, P. Friess, S. Woelfflé, "Vision and Challenges for Realising the Internet of Things," Cluster of European Research Projects on the Internet of Things, European Commission, 2010 , http://www.internet-of-things-

research.eu/pdf/IoT Clusterbook_March 2010.pdf, retrieved $18 / 05 / 2017$.

[14] D. Bandyopadhyay, J. Sen, "Internet of Things - Applications and Challenges in Technology and Standardization," Wireless Personal Communications, vol. 58(1), pp. 49-69, 2011.

[15] A. J. Trappey, C. V. Trappey, U. H. Govindarajan, A. C. Chuang, and J. J. Sun, "A review of essential standards and patent landscapes for the Internet of Things: A key enabler for Industry 4.0," Adv. Eng. Informatics, vol. 33, pp. 208-229, 2017.

[16] Minesoft, "Introduction to Patbase," 2012 https://www.patbase.com/pboverview.pdf, retrieved 10/04/2017.

[17] Postscapes, "Internet of Things (IoT) History," 2016 , https://www.postscapes.com/internet-of-things-history/, retrieved 07/07/2017.

[18] C. H. Song, "Früherkennung von konvergierenden Technologien: Ein neuer Ansatz zur Identifikation attraktiver Innovationsfelder," Wiesbaden: Springer, 2015.

[19] WIPO (World Intellectural Property Organization), "IPC Classifications," 2006, http://www.wipo.int/classifications/ipc/en/ITsupport/Version20060101/t ransformations/pdf/ipcr_catchwordindex en 20060101.pdf, retrieved $12 / 04 / 2017$.

[20] Espacenet, "Classification Search," 2017 https://worldwide.espacenet.com/classification?locale=en_EP, retrieved 10/04/2017.

[21] U. Schmoch, "Concept of a Technology Classification for country comparisons, Final report to the world intellectual property organization," WIPO, $\quad$ pp. $1-15, \quad 2008$, http://www.wipo.int/edocs/mdocs/classifications/en/ipc_ce_41/ipc_ce_4 1_5-annex1.pdf, retrieved 10/04/2017.

[22] Mbaskool, "Top 10 Logistics Companies in World 2015," 2015, http://www.mbaskool.com/fun-corner/top-brand-lists/13971-top-10logistics-companies-in-world-2015.html?start=7, retrieved 15/05/2017.

[23] Gartner, "Gartner's 2014 Hype Cycle for Emerging Technologies Maps the Journey to Digital Business," 2014, http://www.gartner.com/newsroom/id/2819918, retrieved 07/07/2017.

[24] Strategy\&, "The Global ICT-50," 2012 https://www.strategyand.pwc.com/media/file/Strategyand_The-GlobalICT-50.pdf, retrieved 15/04/2017.

[25] M. B. Albert, "The New Innovators: Global Patenting Trends in Five Sectors," Diane Publishing, 2000.

[26] T. H. Davenport, "Process innovation: Reengineering Work through Information Technology," Boston: Harvard Business Press, 1993.

[27] Forbes, "These are the 25 fastest growing Public Tech Companies," 2016, https://www.forbes.com/sites/briansolomon/2016/04/20/these-are-the25-fastest-growing-public-tech-companies/, retrieved 27/07/2017.

[28] S. Racherla, J. Daniel, "IPv6 Introduction and Configuration," 2012, https://www.redbooks.ibm.com/redpapers/pdfs/redp4776.pdf, retrieved 27/07/2017.

[29] M. E. Porter, J. E. Heppelmann, "How smart, connected products are transforming competition," Harvard Business Review, vol. 92(11), pp. 64-88, 2014.

[30] DHL Freight Connections, “Agheera gewinnt den deutschen Telematik Preis 2016,“ 2015, http://dhl-freight-connections.com/de/agheera-winsthe-german-telematics-award-2016-for-its-innovative-tracking-solution/, retrieved 27/07/2017.

[31] Forbes, "New Robotic Solutions For The Warehouse," 2017, https://www.forbes.com/sites/stevebanker/2017/03/07/new-roboticsolutions-for-the-warehouse/\#eed72a76506e, retrieved 27/07/2017.

[32] UPS, "UPS Fleet Telematics System," 2016 , 